The plot of $F_{4}(x)$ (Fig. 3) showed a horizontal line and indicated that only four complexes were involved. As a check on the values for $K_{2}, K_{3}$ and $K_{4}$, the limiting slopes were obtained; which approximate the $K$ values for the next higher complex ${ }^{8}$. The limiting slope for the $F_{1}(x)$ curve was calculated as $3.80 \times 10^{3}$ $\left(K_{2}=4.0 \times 10^{3}\right)$ that for $F_{2}(x)$ curve as $7.90 \times 10^{4}$. $\left(K_{3}=7.0 \times 10^{4}\right)$ and for $F_{3}(x)$ curve as $3.50 \times 10^{6}$ $\left(K_{4}=3.59 \times 10^{6}\right)$.
The present investigation reveals (i) the formation of four complexes $\mathrm{CdA}, \mathrm{CdA}_{2}{ }^{2 \ominus}, \mathrm{CdA}_{3}{ }^{4 \ominus}$ of $\mathrm{Cd}^{2 \oplus}$ with mercapto propionate ion (ii) the $\log K$ values were found to be $\log K_{1}=2.0, \log K_{2}=3.60, \log K_{3}=4.84$ and $\log K_{4}=6.55$ respectively.

Our grateful thanks are due to Ministry of Education, Government of India for grant of Research Scholarship to one of us (K. C. G.), to Evan's Chemetics, Inc. New York, for supplying $\beta$-Mercapto propionic acid as a gift and to principal R. M. Advani for providing research facilities.

\section{Synthesen mit naszierenden Chinonen VI ${ }^{1}$}

\section{Zur Kenntnis des Alizarinchinons}

\section{Hans-Werner Wanzlick, Hans Heidepriem und Carlos Weber-Schilling}

Organisch-Chemisches Institut der Technischen Universität Berlin

(Z. Naturforschg. 24 b, 137-138 [1969]; eingegangen am 23. Oktober 1968)

Die Dehydrierung von Alizarin in Gegenwart von $p$-Toluolsulfinat, Sulfit und Nitrit führt in guten Ausbeuten zu den Alizarin-Derivaten $2-\mathbf{4}^{2}$ :

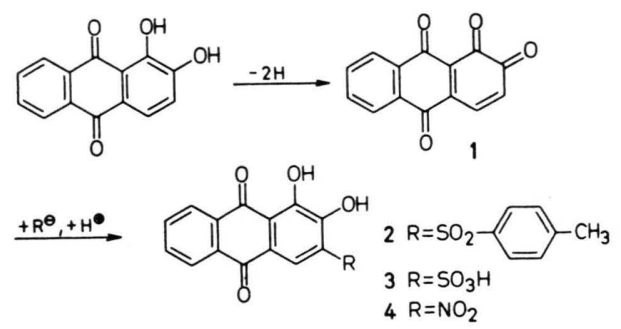

Das durch Dehydrierung des Alizarins entstehende Anthradichinon-(1.2;9.10) (Alizarinchinon) (1), bisher nur in Lösung bekannt ${ }^{3}$, addiert die nucleophilen Partner $\mathrm{R}^{\ominus}$ aus der Polarisation 1 a heraus :

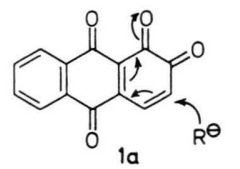<smiles>O=C1C2=C(C(=O)c3ccccc31)C1CCCC1C=C2</smiles>

Die als „normal“ erwartete Addition in 4-Stellung gemäß $1 \mathbf{b}$ findet (aus elektronischen ${ }^{2}$, möglicherweise auch aus sterischen Gründen) nicht statt.

Um zu prüfen, ob diese anomale Addition an in situ erzeugtes Alizarinchinon auch bei C-C-Verknüpfungen eintritt, haben wir Alizarin in Gegenwart des bewährten ${ }^{4}$ Dimedons dehydriert. In befriedigender Ausbeute erhält man hierbei eine leuchtend rote, gut kristallisierte Substanz. Der Verbindung kommt die Formel $6 \mathrm{zu}$. Es findet also auch hier Addition in

1 V. Mitt.: H.-W. Wanzlick u. U. Jahnke, Chem. Ber. 101, 3753 [1968].

2 H.-W. Wanzlick, M. Lehmann-Horchler, S. Mohrmann,

R. Gritzky, H. Heidepriem u. B. Pankow, Angew. Chem. 76, 313 [1964] ; Angew. Chem. internat. Ed. 3, 401 [1964].
3-Stellung statt; die Bildung von $\mathbf{6}$ dürfte über das nicht faßbare Primäraddukt 5 verlaufen:

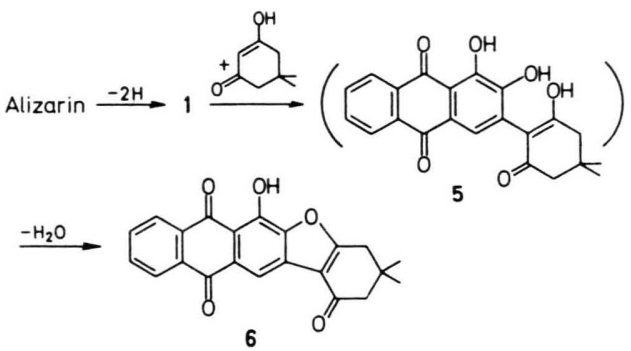

Zum Unterschied von bisher studierten Dimedon-Additionen an naszierende Chinone wird im vorliegenden Falle keine weitere Dehydrierung beobachtet; der Furan-Ringschluß, der sich auch sonst, z. B. bei der Synthese des Wedelolactons ${ }^{4}$, anschließt, kommt hier durch einfache Wasser-Abspaltung zustande.

Die aus Eisessig mit einem Mol Essigsäure kristallisierende rote Verbindung $\left(\lambda_{\max }=495 \mathrm{~nm}(\varepsilon=5500\right.$, Methanol); Alizarin: $\lambda_{\max }=430 \mathrm{~nm}(\varepsilon=5000$, Methanol)) besitzt, wie zu erwarten, nicht mehr die für Alizarin typische Indikatoreigenschaft: Weder Bicarbonat- noch Soda-Zusatz zu alkoholischen 6-Lösungen bewirken die für Alizarin charakteristische Violettfärbung. Erst bei der Behandlung mit Natronlauge tritt allmählich Violettfärbung auf - offenbar infolge Verseifung des „vinylogen Lactons“ 6 zum Anion des Primäradduktes 5. Beim Versuch, 5 durch vorsichtiges Ansäuern der alkalischen Lösung zu isolieren, wird gleich wieder durch spontane Relactonisierung $\mathbf{6}$ erhalten.

Interessante Verhältnisse beobachteten wir bei der Acetylierung: 6 wird durch Acetanhydrid/Pyridin nicht in das erwartete Mono-, sondern in ein überraschend tieffarbiges $D i$-acetat verwandelt:

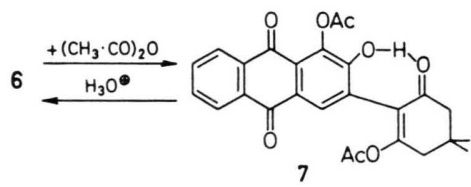

3 O. Dimroth u. E. Schultze, Liebigs Ann. Chem. 411, 345 [1916].

4 H.-W. Wanzlick, R. Gritzky u. H. Heidepriem, Chem. Ber. 96, 305 [1963]. 
Der blauvioletten Farbe des Diacetates wird am besten die Formel 7 gerecht: Durch H-Brückenbindung entsteht ein Chromophor, der dem des Alizarinmonoanions 8 in etwa entspricht $\left(7: \lambda_{\max }=565 \mathrm{~nm}(\varepsilon=\right.$ 4600 , Methanol) ; 8 (Alizarin $\left./ \mathrm{NaHCO}_{3}\right): \lambda_{\max }=540$ $\mathrm{nm}(\varepsilon=7600$, Methanol $))$.

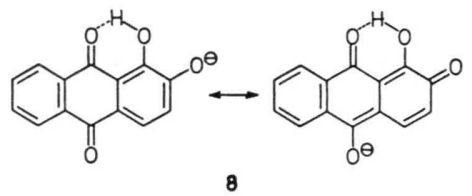

7 wird, wie zu erwarten, leicht zu 6 verseift, z. B. beim Erwärmen in schwach mineralsaurer Lösung.

\section{Beschreibung der Versuche}

Die Analysen verdanken wir der Mikroanalytischen Abteilung unseres Instituts unter Leitung von Frau Dr. U. FAass.

\section{Farbstoff 6}

$\mathrm{Zu}$ einer Lösung von $480 \mathrm{mg}$ Alizarin, $7 \mathrm{~g}$ Dimedon und $10 \mathrm{~g}$ Natriumhydrogencarbonat in $200 \mathrm{~cm}^{3}$ Aceton/Wasser (1:1) gab man die Lösung von 2,64 g Kaliumhexacyanoferrat(III) in $25 \mathrm{~cm}^{3}$ Wasser. Nach einer Stde. säuerte man vorsichtig mit $15 \mathrm{~cm}^{3}$ konz. Salzsäure an. Die dabei ausgeschiedenen roten Flocken wurden abgesaugt und mit Wasser gewaschen. Durch Einengen des Filtrats i. Vak. wurde eine weitere Menge des Farbstoffs erhalten. Gesamtausbeute $350 \mathrm{mg}$ (49\% d. Th.).
Dunkelrote Kristalle (nach mehrmaligem Umkristallisieren aus Eisessig), Schmp. $310^{\circ}$ (Zers.), ab $275^{\circ}$ Sublimation. IR-Spektrum: 1722, 1673, 1622/cm.

$$
\begin{aligned}
& \mathrm{C}_{22} \mathrm{H}_{16} \mathrm{O}_{5} \cdot \mathrm{C}_{2} \mathrm{H}_{4} \mathrm{O}_{2} \quad(420,4) \\
& \text { Ber. C 68,57 H 4,76, } \\
& \text { Gef. C 68,67 H 4,82. }
\end{aligned}
$$

Durch 1-stdg. Erhitzen auf $200^{\circ}$ im Trockenschrank wird 6 essigsäurefrei in Form eines hellroten Kristallpulvers erhalten. Schmp. wie oben. IR-Spektrum: 1673, $1622 / \mathrm{cm}$

$$
\begin{aligned}
& \mathrm{C}_{22} \mathrm{H}_{16} \mathrm{O}_{5} \quad(360,4) \\
& \text { Ber. C 73,30 H 4,44, } \\
& \text { Gef. C 72,76 H 4,33. }
\end{aligned}
$$

Mol.-Gew. 360 (massenspekroskop.* $^{*}$ ).

\section{Diacetat 7}

$100 \mathrm{mg} 6$ wurden in $1 \mathrm{~cm}^{3}$ Pyridin und $1 \mathrm{~cm}^{3}$ Acetanhydrid gelöst. Nach $24 \mathrm{Stdn}$. goß man die Lösung auf Eis. Die ausgefallenen dunkelblau-violetten Kristalle wurden abgesaugt und mit Wasser gewaschen. Ausbeute $90 \mathrm{mg}\left(70 \%\right.$ d. Th.). Schmp. $183-185^{\circ}$ (Zers.) (nach zweimaligem Umkristallisieren aus Aceton/Wasser).

$$
\begin{aligned}
& \mathrm{C}_{26} \mathrm{H}_{22} \mathrm{O}_{8} \quad(462,5) \\
& \text { Ber. C 67,52 H 4,50, } \\
& \text { Gef. C 67,66 H 4,39. }
\end{aligned}
$$

Wir danken der Deutschen Forschungsgemeinschaft, dem Fonds der Chemischen Industrie, der Schering AG und dem Deutschen Akademischen Austauschdienst für erfahrene Hilfe.

* Wir danken Herrn Dr. D. Schumann für die Messung. 